\title{
Postoperative delirium in non-cardiac surgery: systematic review of incidence and risk factors
}

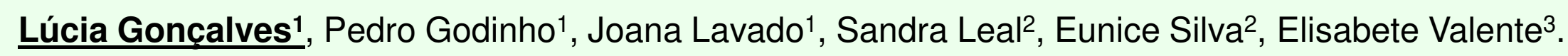

1 - Anaesthesia Resident, 2 - Anaesthesia Assistant, 3 - Head of Department

Department of Anaesthesiology - Centro Hospitalar de Leiria, EPE - Portugal

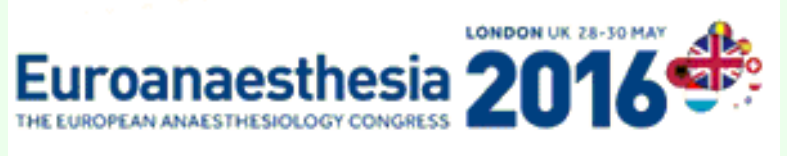

\section{INTRODUCTION/OBJECTIVE}

Postoperative delirium (POD) is a sudden and usually temporary cognitive and emotional disorder, common in older patients submitted to surgery. This is an underdiagnosed, undertreated and still poorly understood condition. This review aims to identify the prevalence and risk factors for POD in order to improve preventive and treatment measures for this condition.

\section{METHODS}

A qualitative systematic review of the literature was conducted in the MEDLINE database. Observational prospective studies published between 2012 and 2015 and assessing the incidence of POD, (determined by the Confusion Assessment Method) and its risk factors were selected. Only English-written studies were included. Study populations included patients aged 65 years or higher, submittec to non-cardiovascular surgery, without known history of POD. Search terms included "postoperative delirium" in the title. The study selection process is described in the diagram below. Risk factors with statistically significant associations reported in at least 3 of the included studies $(p<0.05)$ were assessed

\begin{tabular}{|c|c|}
\hline & \multirow{5}{*}{$\begin{array}{c}\begin{array}{c}\text { Records excluded } \\
\text { ( } \mathbf{n}=\mathbf{6 7})\end{array} \\
13 \text { written in non-English language } \\
28 \text { not studies (editorials, errata, } \\
\text { recommendations, opinion articles) } \\
23 \text { review studies } \\
2 \text { surveys to professionals } \\
1 \text { pre-clinical study }\end{array}$} \\
\hline \multirow{3}{*}{$\begin{array}{l}\text { Records identified } \\
\text { through database } \\
\text { searching and } \\
\text { screened }(n=194)\end{array}$} & \\
\hline & \\
\hline & \\
\hline & \\
\hline & $\begin{array}{l}\text { Full-text articles excluded } \\
\qquad(\mathrm{n}=117)\end{array}$ \\
\hline & 40 - Population criteria \\
\hline Full-text articles & 23 - Other method besides CAM \\
\hline eligibility ( $n=127)$ & 38 - Not observational prospective \\
\hline & proposed objectives \\
\hline 1 & 6 - Study methods not clear \\
\hline $\begin{array}{l}\text { Studies included in } \\
\text { qualitative review } \\
\qquad(n=10)\end{array}$ & \\
\hline
\end{tabular}

\section{RESULTS}

Of a total of 194 screened studies, 10 were considered eligible for inclusion. A total of 4062 patients were included in these studies, with POD rates changing from $8.5 \%$ to $47.5 \%$ (weighted mean: $34.7 \%$ ). Table 1 provides a summarized description of these studies.

Table 2 presents the most common risk factors reported with statistically significant association with POD occurrence, by study.
Table 1. Study overview and postoperative delirium incidence

\begin{tabular}{|c|c|c|c|c|}
\hline $\begin{array}{c}\text { 1st author } \\
\text { (year) }\end{array}$ & N & $\begin{array}{c}\text { Gender } \\
(\mathbf{f} \%)\end{array}$ & Surgery & N(\%) POD \\
\hline Berhends M (2013) & 472 & $47.9 \%$ & Various ${ }^{2}$ & $137(29.0 \%)$ \\
\hline Chu CS (2016) & 544 & $56,4 \%$ & Orthopaedics & $52(9.6 \%)$ \\
\hline Gani H (2013) & 640 & $3.9 \%$ & Urology & $166(25.9 \%)$ \\
\hline Hirsch J (2015) & 540 & $50.6 \%$ & Various 2 & $231(42.8 \%)$ \\
\hline Large MC (2013) & 49 & $18.4 \%$ & Urology & $14(28.6 \%)$ \\
\hline Leung JM (2013) & 581 & $50.3 \%$ & Various & $234(40.3 \%)$ \\
\hline Tai S (2015) & 485 & $0 \%{ }^{3}$ & Urology & $103(21.2 \%)$ \\
\hline Wang J (2015) & 200 & $52.5 \%$ & Orthopaedics & $17(8.5 \%)$ \\
\hline Wu Y (2015) & 130 & $76.2 \%$ & Orthopaedics & $34(26.2 \%)$ \\
\hline Youngblom E (2014) & 421 & $53.4 \%$ & Various & $200(47.5 \%)$ \\
\hline Total sample & $\mathbf{4 0 6 2}$ & Weighted mean & $\mathbf{3 4 . 7 \%}$ \\
\hline
\end{tabular}

1. this study involved prostatectomy surgery. 2. "Various" includes orthopaedic, thoracic, abdominal and other miscellaneous procedures. 3. "Various" includes orthopaedic, uro/ gynecological, vascular and other miscellaneous procedures.

Table 2. Statistical significant associations found between incidence of postoperative delirium and most commonly reported risk factors

\begin{tabular}{|c|c|c|c|c|c|}
\hline 1st author & Ageing & $\begin{array}{l}\text { Cognitive/ } \\
\text { affective } \\
\text { impairment }\end{array}$ & $\begin{array}{l}\text { Functional } \\
\text { dependence }\end{array}$ & $\begin{array}{c}\text { Perioperative } \\
\text { anaemia }\end{array}$ & $\begin{array}{l}\text { Intraop } \\
\text { blood loss/ } \\
\text { transfusion }\end{array}$ \\
\hline Berhends M & $\checkmark$ & $\checkmark$ & ne & $\checkmark$ & $\checkmark$ \\
\hline Chu CS & $\checkmark$ & $\checkmark$ & $\checkmark$ & $x$ & $\checkmark$ \\
\hline Gani $\mathrm{H}$ & $\checkmark$ & ne & ne & $\checkmark$ & $\checkmark$ \\
\hline Hirsch J & $\checkmark$ & $\checkmark$ & $x$ & $\checkmark$ & $\checkmark$ \\
\hline Large MC & $\checkmark$ & $\checkmark$ & $x$ & $x$ & $x$ \\
\hline Leung JM & ne & $\checkmark$ & $\checkmark$ & $\checkmark$ & ne \\
\hline Tai S & $\checkmark$ & $\checkmark$ & $\checkmark$ & ne & ne \\
\hline Wang J & $\checkmark$ & $\checkmark$ & ne & ne & $\checkmark$ \\
\hline Wu Y & ne & $\checkmark$ & ne & ne & ne \\
\hline Youngblom E & ne & $\checkmark$ & ne & ne & ne \\
\hline
\end{tabular}

$\checkmark$ - Statistically significant association found.

X- Statistically significant association assessed but not found.

ne - association not evaluated in this study.

\section{DISCUSSION}

Included studies addressed several surgical specialties, with Orthopaedic and Urologic surgery being the most frequent. Apart from two exceptions, the incidence of POD was higher than $20 \%$ in all studies, with a weighted mean incidence of $34.7 \%$, thus demonstrating the significant risk of this condition in older patients. Several key demographic preoperative and intraoperative variables were also identified as common risk factors in several of the included studies. The anaesthetist should considered these variables when assessing the risk for POD development in older patients, in the preoperative clinical setting 